\title{
Chronic Obstructive Pulmonary Disease Rehabilitation and 6-Minutes Walk Test Revisited
}

\author{
Laurent P. Nicod \\ Lausanne University, Lausanne, Switzerland
}

Regular physical activity has been shown to reduce hospital admission and mortality of chronic obstructive pulmonary disease (COPD) [1]. If patients can increase their physical activity, their prognosis has been shown to improve in large cohort studies. Data even suggest that it is important to assess and encourage regular physical activity at the earliest stages of COPD in order to maintain a physical activity level that is as high as possible, as this is associated with a better prognosis [2].

This functional capacity can be evaluated by various methods as the 6 -min walk test (6MWT), the shuttle walk test (SWT), or the cardiopulmonary exercise test (CPET). The $6 \mathrm{MWT}$ is widespread because of its simplicity, monitoring the distances walked during field walking and the blood O2 saturation. The 6 MWT has good correlates with symptoms limiting cardiopulmonary exercise either for COPD or interstitial lung diseases and can well assess outcome following rehabilitation [3]. Relationships between field walking and incremental cycle ergometry in COPD have been tested leading to various and discrepant findings at this point. A strong agreement between peak work rate was measured on the CPET and that estimated from either the 6 MWT or the incremental shuttle walk test [4]. Troosters et al. [5] compared the physiological

\section{karger@karger.com www.karger.com/res

responses of 20 patients with COPD during an encouraged 6MWT and maximal-cycle ergometer exercise testing. They demonstrated that 6 MWT and peak cycling showed identical VO2 responses and similar heart rate (HR) responses. As the VCO2 and ventilatory response were found to be lower during walking than at peak cycling, it was concluded that the 6 MWT should be referred to as submaximal. Since most activities of daily living are performed in a nonincremental fashion and at a submaximal level of exertion, the $6 \mathrm{MWT}$ is thought by most to be representative of daily life activities and can therefore accurately reflect the functional capacity of patients. Accordingly, Van Helvoort et al. [6] found that VO2 and HR were lower after the $6 \mathrm{MWT}$ than at peak cycling confirming this concept. Nevertheless, Hill et al. [7] compared the peak and submaximal cardiopulmonary responses to the 6 MWT with a ramp-cycle ergometer test in 24 patients with moderate COPD. In accordance with the results of Troosters et al. [5], they demonstrated that the 6 MWT elicited a similar VO2 peak rate and HR as a cycle ergometer test. Turner et al. [7] also observed similar peak exercise responses achieved in the $6 \mathrm{MWT}$ and CPET in 20 patients with moderate to severe COPD. 
In the recent issue of Respiration, Vonbank et al. [8] assess the agreement between oxygen uptake measured during the 6 MWT by a portable equipment and similarly during incremental cycle exercise in COPD patients with GOLD I-IV. Interestingly, they provide an explanation for the discrepancies mentioned above, as indeed no significant correlation between $\mathrm{VO} 2$ measurements could be found between both tests in COPD GOLD I and II $(r=0.17)$, whereas the VO2 significantly correlated in patients with COPD stages III and IV $(r=0.7)$. This is probably the essential explanation and should be taken into account, as early stages of COPD seem to be able to perform much better during CPET than $6 \mathrm{MWT}$. In contrast, in COPD with advanced diseases in particular with hypercapnia, the $6 \mathrm{MWT}$ correlates well with CPET findings and as such is a very good test, sufficient to predict outcome in this type of population with advanced respiratory disease [9]. On the other hand, CPET is certainly more appropriate to find the various cardiovascular or respiratory limitations and their improvements along early and sustained rehabilitation protocols. Thus, exercise therapy should be part of rehabilitation programs that aim to improve both quality of life and physiological status in patients with mild or moderate COPD. CPET should have a central role in the early assessment of most candidates before the rehabilitation program [10].

\section{Conflict of Interest Statement}

The author has no conflict of interest.

\section{References}

1 Garcia-Aymerich J, Lange $P$, Benet $M$, Schnohr P, Antó JM. Regular physical activity reduces hospital admission and mortality in chronic obstructive pulmonary disease: a population based cohort study. Thorax. 2006 Sep;61(9):772-8.

2 Vaes AW, Garcia-Aymerich J, Marott JL, Benet M, Groenen MT, Schnohr P, et al. Changes in physical activity and all-cause mortality in COPD. Eur Respir J. 2014 Nov; 44(5):1199-209.

3 Clini EM, Crisafulli E. Exercise capacity as a pulmonary rehabilitation outcome. Respiration. 2009;77(2):121-8.

4 Luxton N, Alison JA, Wu J, Mackey MG. Relationship between field walking tests and incremental cycle ergometry in COPD. Respirology. 2008 Nov;13(6):856-62.
5 Troosters T, Vilaro J, Rabinovich R, Casas A, Barberà JA, Rodriguez-Roisin R, et al. Physiological responses to the 6-min walk test in patients with chronic obstructive pulmonary disease. Eur Respir J. 2002 Sep;20(3):564-9.

6 van Helvoort HA, Heijdra YF, de Boer RC, Swinkels A, Thijs HM, Dekhuijzen PN. Sixminute walking-induced systemic inflammation and oxidative stress in muscle-wasted COPD patients. Chest. 2007 Feb;131(2):43945.

7 Turner SE, Eastwood PR, Cecins NM, Hillman DR, Jenkins SC. Physiologic responses to incremental and self-paced exercise in COPD: a comparison of three tests. Chest. 2004 Sep; 126(3):766-73.
8 Vonbank K, Marzluf B, Knötig M, Funk GC Agreement between Cardiopulmonary Exercise Test and Modified 6-Min Walk Test in determining oxygen uptake in COPD patients with different stages. Respiration. 2020, DOI: 505856.

9 Budweiser S, Heidtkamp F, Jörres RA, Heinemann F, Arzt M, Schroll S, et al. Predictive significance of the six-minute walk distance for long-term survival in chronic hypercapnic respiratory failure. Respiration. 2008;75(4): $418-26$.

10 Clinical exercise testing with reference to lung diseases: indications, standardization and interpretation strategies. ERS Task Force on Standardization of Clinical Exercise Testing. European Respiratory Society. Eur Respir J. 1997 Nov; 10(11):2662-89. 